\title{
Posicionamento de Pontos de Acesso Sem-fio para Segurança de Visitantes em Parques Florestais
}

\author{
Gabriel F. C. de Queiroz ${ }^{1,2}$, Ana Elisa Ferreira ${ }^{1,3}$, Fernando M. Ortiz ${ }^{1}$, \\ Rodrigo de S. Couto ${ }^{4}$, Luís Henrique M. K. Costa ${ }^{1}$
}

${ }^{1}$ Grupo de Teleinformática e Automação (GTA)

COPPE - Programa de Engenharia Elétrica - UFRJ

${ }^{2}$ DEPES/DETEL - Unidade: Maracanã

Centro Federal de Educação Tecnológica Celso Suckow da Fonseca (CEFET/RJ)

${ }^{3}$ Engenharia de Computação - Unidade: Petrópolis

Centro Federal de Educação Tecnológica Celso Suckow da Fonseca (CEFET/RJ)

${ }^{4}$ Universidade do Estado do Rio de Janeiro - PEL - DETEL/FEN

\{gqueiroz, ferreira, fmolano, luish\}@gta.ufrj.br

rodrigo.couto@uerj.br

\begin{abstract}
The deployment of wireless networks in tracks of forest parks, such as those of the Atlantic Forest, has challenges due to high humidity and dense vegetation. Nevertheless, this type of communication is important for the safety of the park visitors, assisting in natural disaster monitoring and people tracking. This paper formulates an integer linear programming problem for the placement of access points in forest tracks. In order to obtain the parameters of the problem, range measurements of wireless technologies are carried out, and information from the Petrópolis-Teresópolis crossing is used. Results show that it is possible to maintain $79 \%$ of coverage even when reducing in $47 \%$ the number of access points required.
\end{abstract}

Resumo. A implantação de redes sem-fio em trilhas de parques florestais, como os da Mata Atlântica, possui desafios devido à alta umidade e vegetação densa. Entretanto, esse tipo de comunicação é importante para a segurança dos visitantes do parque, auxiliando o monitoramento de acidentes naturais e o rastreamento de pessoas. Este artigo formula um problema de programação linear inteira para posicionamento de pontos de acesso em trilhas florestais. Para obter os parâmetros do problema, realizam-se medições do alcance de tecnologias sem-fio e utilizam-se informações da travessia Petrópolis-Teresópolis. Os resultados mostram que é possível manter uma cobertura de 79\% mesmo com uma redução de $47 \%$ da quantidade de pontos de acesso necessários.

\section{Introdução}

As Unidades de Conservação (UC) ambiental, como parques e reservas florestais, cumprem funções importantes na preservação do meio ambiente, na promoção da educação ambiental e como forma de turismo e integração da sociedade com um ambiente natural. Assim, as atividades de ecoturismo estão atraindo cada vez mais participantes. 
Infelizmente, aumentam também os casos de visitantes de parques naturais que vivenciam situações de risco. Entre os riscos mais graves estão o de se perder em um ambiente desconhecido e a ocorrência de desastres naturais, como cabeças d'água e incêndios.

Uma forma de reduzir os riscos dos visitantes é acompanhar sua localização e atividades. Isso pode ser realizado implantando-se redes sem-fio nos parques florestais [Loureiro et al. 2003]. Essas redes também podem prover informações e alertas para os visitantes. Entretanto, o ambiente de floresta é peculiar e traz uma série de desafios ao projeto. Isso é causado pela dificuldade de propagação da onda eletromagnética e pela limitação de alimentação elétrica. Busca-se manter o projeto simples e barato para facilitar a sua implementação em diferentes UCs. A meta é maximizar a cobertura das redes sem-fio, todavia reduzindo a infraestrutura. Dessa forma, reduz-se o custo do projeto e também os impactos ambientais que possam ser causados à fauna e à flora.

Dado o exposto, este trabalho formula um problema para posicionamento de pontos de acesso sem-fio, também denominados de totens. Os totens proveem a infraestrutura de acesso para os terminais dos usuários e se conectam ao servidor de monitoração de visitantes, diretamente ou usando múltiplos saltos através de outros totens. A formulação de um problema de Programação Linear Inteira (Integer Linear Programming, ILP) é feita para resolver o problema proposto. Para isso, é desenvolvida uma modelagem matricial de cubos para mapear a localização tridimensional do totem, considerando a cobertura do sinal nas trilhas e a atenuação causada pela diferença de altura entre as antenas dos totens. Para solução do modelo proposto, utilizam-se parâmetros reais. Assim, testes de campo são realizados com diferentes tecnologias de comunicação sem-fio, buscando identificar aquela com melhor desempenho para a propagação no ambiente de floresta, que maximiza a área de cobertura e, consequentemente, reduz a quantidade de totens necessários. As principais contribuições deste trabalho são:

- a formulação do problema genérico de posicionamento de totens de comunicação, aplicável em ambientes florestais;

- a proposta de um método de eliminação de cubos impossíveis, que permite reduzir a complexidade do problema formulado; e

- a análise do impacto de diferentes tecnologias de comunicação em ambientes florestais, a partir da solução do problema.

Os resultados apontam que é possível manter a cobertura de $79 \%$ dos cubos da trilha ao utilizar 300 totens ZigBee, $47 \%$ a menos que o número de totens necessários para uma cobertura completa. Da mesma forma, com 200 totens LoRa, já se torna possível cobrir $100 \%$ do percurso da trilha. O artigo está organizado da seguinte forma: na Seção 2, descrevem-se as tecnologias de redes sem-fio consideradas; a Seção 3 detalhada o caso em estudo e os seus desafios. O problema de otimização é formulado na Seção 4, enquanto a Seção 5 apresenta os resultados obtidos. Em seguida, são discutidos os trabalhos relacionados na Seção 6. Finalmente, a Seção 7 conclui o artigo e aponta as direções futuras.

\section{Tecnologias de Comunicação Analisadas}

As tecnologias são avaliadas para atender tanto a comunicação entre totens como entre totem e terminal de usuário, uma pulseira a ser usada durante a estadia na UC. Esta abordagem permite monitorar a localização para rastrear visitantes perdidos sem perturbar a experiência no ambiente natural. A escolha das tecnologias baseia-se nas restrições do 
ambiente e do terminal dos visitantes. Dentre as restrições do ambiente, existem a forte atenuação do sinal causada pela vegetação e a dificuldade de energia. Para os terminais é importante que possuam baixo consumo de energia, sejam pequenos, de baixo custo e de fácil substituição. Assim, avalia-se o uso de comunicação via LoRa e ZigBee.

\subsection{Descrição}

O LoRa, solução proprietária da Semtech, é uma das principais tecnologia usadas em redes de baixo consumo e longa distância (Low Power Wide Area Network, LPWAN). Utiliza modulação do tipo Chirp Spread Spectrum (CSS) e correção de erro (Forward Error Correction, FEC). Pode operar mesmo com uma baixa relação sinal ruído [Semtech Corporation 2015]. Os nós ajustam a potência de saída e a taxa de transmissão para obter melhor alcance e economia de energia. As taxas de dados variam com as restrições regulatórias de cada região e o fator de espalhamento (Spread Factor, SF) utilizado. Um maior fator de espalhamento melhora a relação sinal ruído, porém aumenta o tempo de transmissão [Bor et al. 2016]. O LoRa opera nas faixas de frequência de 433, 868, e $900 \mathrm{MHz}$ ISM, com taxas de dados variando de 0,3 a $50 \mathrm{kbps}$ e alcance entre 2 e $5 \mathrm{~km}$ (área urbana) e $15 \mathrm{~km}$ (área suburbana). Seu uso em múltiplos saltos é proposto no protocolo LoRaBlink [Bor et al. 2016], que de forma similar ao protocolo LoRaWAN [Marais et al. 2017], usa a interface física do LoRa. Além do LoRaBlink, o uso de LoRa em topologias com múltiplos saltos é apresentado em [Van de Velde 2017] através de um nó encaminhador e em [Liao et al. 2017], usando transmissão concorrente.

O ZigBee é uma tecnologia de área pessoal (Wireless Personal Area Network, WPAN) para aplicações com baixa taxa de transmissão e baixo consumo de energia, baseada no padrão IEEE 802.15.4. Possui alcance de até $250 \mathrm{~m}$ com taxa de dados até $250 \mathrm{kbps}$. Pode operar nas faixas de $868 \mathrm{MHz}, 902 \mathrm{MHz}$ e 2,4 GHz. No Brasil, é usada a canalização na faixa 2,4 GHz, com 16 canais com $2 \mathrm{MHz}$ de largura e modulação por deslocamento de fase em quadratura (Quadrature Phase Shift Keying, QPSK) e espalhamento espectral por sequência direta (Direct Sequence Spread Spectrum, DSSS). O ZigBee usa dois tipos de terminais diferentes, com funções reduzidas (Reduced Function Device, RFD), e com função completa (Full Function Device, FFD), que funciona como coordenador da rede [Semtech Corporation 2012]. Com estes dispositivos, é possível criar diferentes topologias, inclusive uma rede em malha. Essa flexibilidade é desejável para o projeto, assim como a comunicação em múltiplos saltos.

\subsection{Medições Experimentais}

Para a avaliação de ambas as tecnologias, são realizadas medições de campo na sede de Teresópolis do Parque Nacional da Serra dos Órgãos (PARNASO), em trilhas com vegetação densa. Essa vegetação afeta gravemente a propagação. Adicionalmente, as medições foram realizadas em diferentes condições atmosféricas, porém, foram priorizadas as medições feitas sob chuva. A escolha das piores condições de testes deve-se ao fato de que o desaparecimento de pessoas ocorre com mais frequência em dias de tempo ruim.

São utilizados dois nós, um móvel e um fixo, responsáveis, respectivamente, pelo envio e recepção dos pacotes de teste. Os testes são feitos com o envio de 200 pacotes, repetidos três vezes para cada ponto. Os pontos de medição foram espaçados entre 0 e $125 \mathrm{~m}$, com um incremento de $25 \mathrm{~m}$ a cada medição. Na Tabela 1, estão descritos os equipamentos dos nós LoRa usados nos testes, configurados com potência de transmissão de 
$14 \mathrm{dBm}$, fator de espalhamento SF7, faixa de frequência de $915 \mathrm{MHz}$ e largura de banda de $500 \mathrm{kHz}$. Foi usada verificação de redundância cíclica (Cyclic Redundancy Check, CRC) de 4/5 e antenas com ganho de $7 \mathrm{dBi}$. Para os nós ZigBee, descritos na Tabela 2, a potência de transmissão é $0 \mathrm{dBm}$, a potência do Xbee Series 1 . É usado o canal de $2,410 \mathrm{GHz}$ com largura de banda de $2 \mathrm{MHz}$ e antena interna ao módulo.

Tabela 1. Equipamentos usados para os nós LoRa.

\begin{tabular}{|l||l||l|}
\hline \multicolumn{1}{|l||}{ Módulo } & Equipamento & Fabricante \\
\hline Controlador nó móvel & Arduino Uno R3 & Arduino \\
\hline Controlador nó fixo & Arduino Yún & Arduino \\
\hline Placa GPS & U-blox NEO-6M & DuinoPeak \\
\hline Placa LoRa & RF96 & Dragino \\
\hline Antena & Antena 7dBi & D-Link \\
\hline Sensores & $\begin{array}{l}\text { Sensor de Umidade DHT22 } \\
\text { Sensor de Temperatura DHT22 }\end{array}$ & $\begin{array}{l}\text { Aosong } \\
\text { Aosong }\end{array}$ \\
\hline
\end{tabular}

Tabela 2. Equipamentos usados para os nós ZigBee.

\begin{tabular}{|l||l||l|}
\hline Módulo & Equipamento & Fabricante \\
\hline Controlador nó móvel e fixo & Arduino Uno R3 & Arduino \\
\hline Placa GPS & U-blox NEO-6M & DuinoPeak \\
\hline Placa XBee & Shield XBee & ArduinoxBee \\
\hline XBee & XBee Series 1 & MaxStream \\
\hline \multirow{2}{*}{ Sensores } & $\begin{array}{l}\text { Sensor de Umidade DHT11 } \\
\text { Sensor de Temperatura DHT11 }\end{array}$ & $\begin{array}{l}\text { DfRobot } \\
\text { DfRobot }\end{array}$ \\
\hline
\end{tabular}

Conforme esperado, o desempenho de ambas as tecnologias foi muito prejudicado devido à atenuação causada pela vegetação e pela chuva, reduzindo a área de cobertura prevista de $2 \mathrm{Km}$ para $125 \mathrm{~m}$ no caso do LoRa, com potência de recepção de $126,08 \mathrm{dBm}$. Para o ZigBee a redução foi de $250 \mathrm{~m}$ para $50 \mathrm{~m}$, com potência de recepção de $-26,99 \mathrm{dBm}$. A taxa de perda de pacotes também foi elevada chegando a 14,10\% acima de $100 \mathrm{~m}$ para o LoRa e 15,09\% acima de $25 \mathrm{~m}$ para o ZigBee. Esses valores demonstram a dificuldade encontrada para a propagação em ambiente de floresta, como descrito em [Figueiredo et al. 2009], agravada pela condição meteorológica.

\section{Estudo de Caso}

O estudo de caso deste artigo é a aplicação da otimização para o posicionamento dos totens na trilha da travessia entre as cidades de Petrópolis e Teresópolis através do PARNASO. A trilha, com cerca de $28 \mathrm{~km}$ de extensão, é percorrida em três dias de caminhada, com pernoite em dois abrigos de montanha. O procedimento do PARNASO exige que, antes do início da caminhada, seja assinado o Termo de Conhecimento de Riscos e Normas. O termo, entregue nas portarias do parque, é importante para o registro da entrada e saída dos visitantes. O horário de entrada e o tempo estimado de percurso são usados para prever a chegada do visitante nos abrigos. Assim, no caso de algum visitante se perder, essa ocorrência só é percebida à noite e possivelmente depois de várias horas, dificultando o resgate. A Figura 1 ilustra o percurso da travessia Petrópolis-Teresópolis. Os pontos indicados dentro de círculos azuis são as localizações dos dois abrigos para pernoite. $\mathrm{O}$ trecho entre os abrigos é o mais propenso à ocorrência de desaparecimentos. 


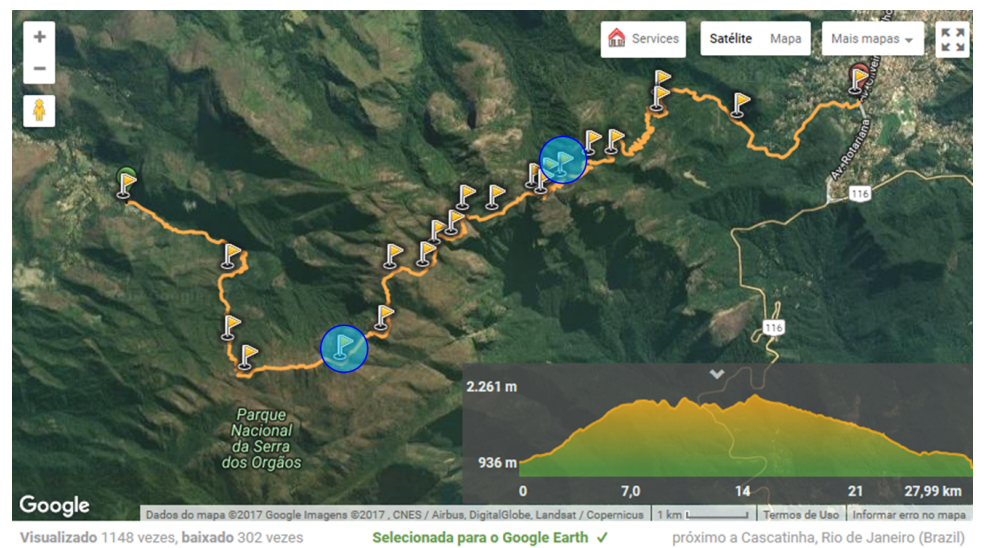

Figura 1. Percurso da travessia Petrópolis-Teresópolis.

\section{Modelagem do Problema de Otimização}

Para prover uma infraestrutura de comunicação ao longo da travessia, totens contendo módulos das tecnologias disponíveis, apresentados na Seção 2, serão instalados em pontos específicos na extensão do trajeto. Os totens proveem conectividade dentro de sua região de cobertura tridimensional. A variação de altitude ao longo da trilha impacta a transmissão e a recepção do sinal pois a irradiação das antenas no plano de azimute pode ser muito diferente da irradiação no plano de elevação [Stutzman and Thiele 2012]. Isso justifica levar em consideração a altura dos totens e do terreno em um mapeamento tridimensional da região descrita pela trilha.

O mapeamento em uma matriz tridimensional é detalhado a seguir. Em resumo, a região de cobertura dos totens é definida com base na noção de cubos. Para lidar com o grande número de cubos no mapeamento, um método para eliminação de cubos "impossíveis" é desenvolvido. Então, a formulação do problema de ILP é discutida.

\subsection{Mapeamento em Cubos}

Para definir os pontos de posicionamento ótimos dos totens de comunicação, a região tridimensional descrita pela trilha é dividida em cubos. As condições desafiadoras de propagação na mata, devido à elevada atenuação causada pela vegetação densa, do solo ao topo das árvores, e a grande variação de altitude, justificam a abordagem baseada em cubos para alcançar um posicionamento condizente com o cenário real.

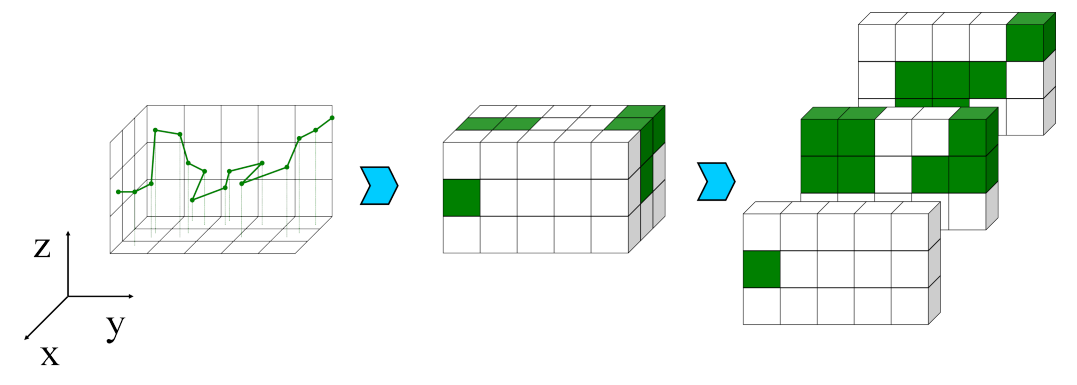

Figura 2. Mapeamento em cubos de um percurso dentro de uma região.

O mapeamento em cubos é realizado tomando os valores máximos e mínimos de latitude, longitude e altitude da travessia que descrevem as dimensões de um paralelepípedo 
que contém a linha da trilha. Em seguida, a região é dividida em cubos, que são mapeados em coordenadas através da definição do valor de lado do cubo. Assim, os pontos de latitude, longitude e altitude resultam em índices $x, y \mathrm{e} z$ da matriz tridimensional, respectivamente. A medida do lado do cubo é ajustada levando-se em consideração o alcance de transmissão do totem, que é discutida em mais detalhes na Subseção 4.2. Valores menores de lado do cubo trazem uma granularidade mais fina ao escolher a posição dos totens, mas aumentam a quantidade de cubos na matriz e, portanto, a complexidade do problema. $\mathrm{O}$ processo de como é feita a escolha da granularidade é tratado na Subseção 5.1.

Com a matriz tridimensional definida, é possível mapear pontos de interesse em seu interior. Tais pontos são definidos como locais nos quais se deseja ter conectividade e capacidade de comunicação, como pontos comuns de visitação ou o próprio percurso da trilha. A Figura 2 ilustra esse processo. O percurso em verde atravessa parte dos cubos contidos nela. Esses cubos são destacados e representam pontos de interesse dentro da matriz. À direita, é possível uma visualização melhor dos cubos na matriz de exemplo. O mapeamento em cubos é feito de forma genérica e pode utilizar qualquer trilha ou região para mapear pontos de interesse. Da mesma forma, algumas regiões podem ter maior ou menor interesse do que outras e isso também é configurável na modelagem adotada.

\subsection{Região de Cobertura}

A região de cobertura de um totem de comunicação também é definida em cubos, obedecendo os valores de alcance obtidos nos testes práticos realizados no PARNASO. A partir de um cubo central, no qual o totem está localizado, a região de cobertura é definida nas três direções: latitude, longitude e altitude, representadas pelos índices $x, y$ e $z$, respectivamente. A região de cobertura corresponde aos cubos próximos a um totem em que a comunicação é viável. A Figura 3 mostra um exemplo de região de cobertura, em verde claro, para o totem posicionado no cubo verde escuro. No exemplo, o alcance em cubos nas três direções é de $l_{x}=1, l_{y}=2$ e $l_{z}=1$. Isso significa que a região de cobertura se estende até um cubo a partir do totem tanto no eixo $x$ quanto no eixo $z$ e até dois cubos à esquerda e à direita do totem, no eixo $y$.

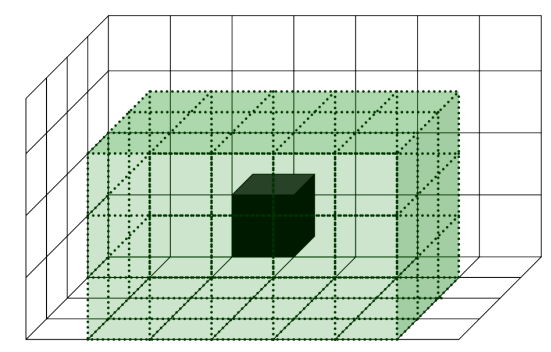

Figura 3. Definição da região de cobertura iluminada por um totem.

Ainda com relação à Figura 3, é possível deduzir a fórmula para o número de cubos cobertos por um totem com base na extensão do alcance nas três direções $x, y \mathrm{e} z$. A Equação 1 mostra o número máximo de cubos $\mathcal{A}_{\max }$ iluminados por um totem. Esse valor é um máximo, pois é possível que haja um número de cubos dentro da região de cobertura $\mathcal{A}_{x, y, z}<\mathcal{A}_{\text {max }}$. Isso ocorre, por exemplo, quando o totem é posicionado muito próximo das bordas da matriz tridimensional, de modo que alguns dos cubos iluminados 
estejam fora da matriz.

$$
\mathcal{A}_{\max }=\left(2 l_{x}+1\right) \cdot\left(2 l_{y}+1\right) \cdot\left(2 l_{z}+1\right) .
$$

\subsection{Método de Eliminação de Cubos Impossíveis}

Ao realizar o mapeamento em cubos, a diferença entre a altitude mínima e a máxima do paralelepípedo incide na criação de cubos tanto subterrâneos quanto aéreos. Esses cubos são pontos dentro da matriz que se situam abaixo do nível de chão e em pleno ar, no caso dos cubos subterrâneos e dos cubos aéreos, respectivamente. Tais cubos em que o posicionamento de um totem de comunicação é claramente impossível ou inviável em nada contribuem para o problema de otimização, tornando-o computacionalmente mais difícil de forma desnecessária.
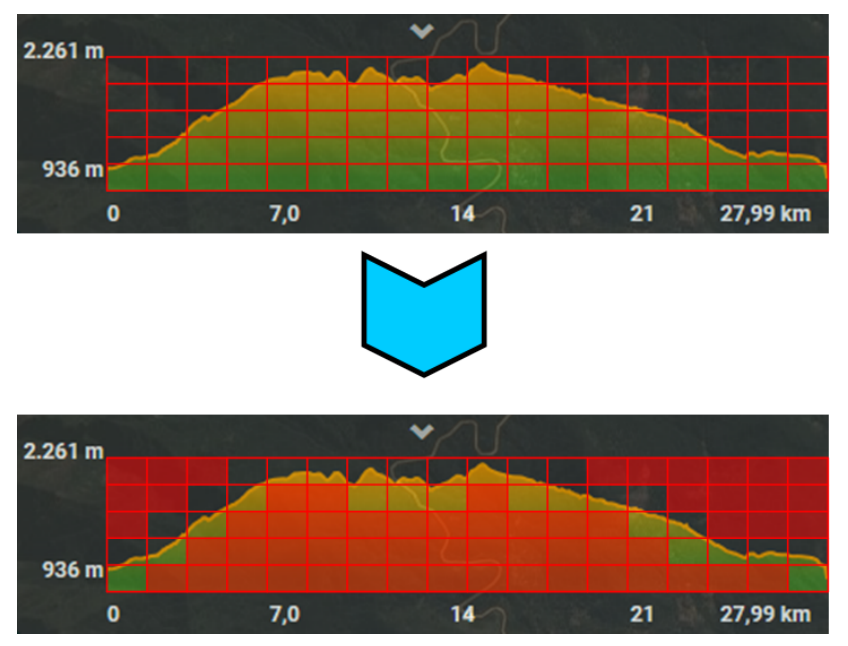

Figura 4. Exemplo de eliminação de cubos impossíveis.

O método de eliminação de cubos impossíveis realiza um pré-processamento em que os dados de entrada da matriz tridimensional são tratados de forma a eliminar cubos subterrâneos ou aéreos. Em seguida, apenas os cubos necessários são entregues ao CPLEX $^{1}$ para solucionar o problema ILP. O CPLEX é uma ferramenta de otimização amplamente conhecida, que foi desenvolvida pela IBM para solucionar problemas de programação linear, inteira e quadrática. A Figura 4 ilustra um exemplo de eliminação de cubos. Na parte de cima, a matriz é gerada e os cubos são definidos. Porém, a maioria dos cubos é composta por pontos subterrâneos ou aéreos devido à variação de altitude. Os cubos que não possuem nível de chão, isto é, cuja altitude não corresponde à altitude do local físico, são eliminados da matriz. Os cubos removidos estão destacados em vermelho.

A partir dos dados de satélite obtidos com a ferramenta GPS Visualizer ${ }^{2}$, a altitude da superfície da Terra é obtida para cada par de latitude e longitude. A matriz tridimensional completa é gerada como mostrado na Figura 2. Para cada par de índices $x$ e $y$ da matriz, os índices $z$ são confrontados com os pontos de chão dos dados de satélite. Se o cubo possuir um índice $z$ diferente do nível de solo, ele é removido da matriz e considerado impossível, seja subterrâneo ou aéreo. É possível que haja mais de um valor de $z$

\footnotetext{
${ }^{1}$ IBM CPLEX: https://www-01.ibm.com/software/commerce/optimization/cplex-optimizer/

${ }^{2}$ GPS Visualizer: http://www.gpsvisualizer.com
} 
viável para um mesmo par $x y$, como mostrado na Figura 4. Nesse caso, todos os cubos viáveis de uma coluna $x y$ são mantidos na matriz. Após verificar cada par $x y$, a matriz reduzida é formada e entregue ao solver. O fluxograma da Figura 5 ilustra o procedimento para eliminação de cubos impossíveis.

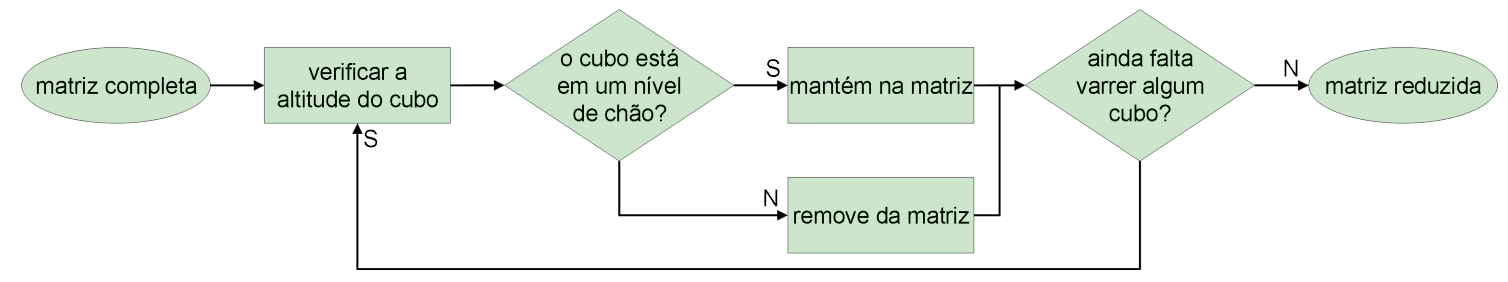

Figura 5. Fluxograma de geração da matriz reduzida.

\subsection{Formulação Matemática}

Para resolver o problema de otimização, foi formulado um problema de ILP cujo objetivo é maximizar o interesse obtido ao iluminar os cubos de uma matriz tridimensional. Cada cubo é considerado iluminado se sua região estiver dentro da cobertura do sinal emitido por um totem nas redondezas. Já o interesse é um parâmetro associado a cada cubo nas coordenadas $x, y$ e $z$, definindo a importância daquela região. Por exemplo, cubos nos quais se encontrem trechos da trilha ou pontos comuns de visitação ou de desaparecimento de pessoas, podem ser considerados de maior interesse do que pontos muito distantes ou fora da área do parque. A Equação 2 mostra a função objetivo. A descrição dos conjuntos, parâmetros e variáveis usados na modelagem do problema é apresentada na Tabela 3.

$$
\operatorname{maximizar} \sum_{x \in \mathcal{X}} \sum_{y \in \mathcal{Y}} \sum_{z \in \mathcal{Z}} \mathcal{I}_{x, y, z} \cdot c_{x, y, z}
$$

As Inequações 3 a 5 representam as restrições do problema. A Inequação 3 define que o número total de totens posicionados não pode exceder o número $\mathcal{N}$ de totens disponíveis para o posicionamento. A Inequação 4 indica que, se um determinado cubo está dentro da região de cobertura de algum totem, isto é, tem conectividade, então há pelo menos um totem em algum dos cubos vizinhos capaz de alcançá-lo. O parâmetro $\mathcal{A}_{x, y, z}$ representa o número de cubos alcançáveis por um totem, que são os cubos em verde claro na Figura 3. O número máximo de cubos que um totem pode iluminar é dado pela Equação 1. No entanto, o número de cubos dentro da região de cobertura de um totem não é sempre $\mathcal{A}_{\text {max }}$. Por exemplo, se um totem estiver em um vértice da matriz tridimensional, $\mathcal{A}_{x, y, z}<\mathcal{A}_{\text {max }}$, pois parte da região de cobertura nesse caso se estende para fora da matriz tridimensional, então a região de cobertura fica limitada aos cubos que estão no interior da matriz. Já a Inequação 5 fornece conectividade de múltiplos saltos entre os totens, fazendo com que haja pelo menos $\mathcal{R}$ totens vizinhos dentro da região de cobertura de cada totem.

$$
\begin{gathered}
\text { sujeito a } \sum_{x \in \mathcal{X}} \sum_{y \in \mathcal{Y}} \sum_{z \in \mathcal{Z}} t_{x, y, z} \leq \mathcal{N} \\
0 \leq \mathcal{A}_{x, y, z} \cdot c_{x, y, z}-\sum_{\substack{u=x-l_{x} \\
u \in \mathcal{X}}}^{x+l_{x}} \sum_{\substack{v=y-l_{y} \\
v \in \mathcal{Y}}}^{y+l_{y}} \sum_{\substack{w=z-l_{z} \\
w \in \mathcal{Z}}}^{z+l_{z}} t_{u, v, w} \leq \mathcal{A}_{x, y, z}-1 \quad \forall x \in \mathcal{X}, y \in \mathcal{Y}, z \in \mathcal{Z}
\end{gathered}
$$




$$
\mathcal{R} \cdot t_{x, y, z} \leq \sum_{\substack{u=x-l_{x} \\ u \in \mathcal{X}}}^{x+l_{x}} \sum_{\substack{v=y-l_{y} \\ v \in \mathcal{Y}}}^{y+l_{y}} \sum_{\substack{w=z-l_{z} \\ w \in \mathcal{Z}}}^{z+l_{z}} t_{u, v, w}-t_{x, y, z} \quad \forall x \in \mathcal{X}, y \in \mathcal{Y}, z \in \mathcal{Z}
$$

Tabela 3. Notações utilizadas no problema.

\begin{tabular}{|c||l||c|}
\hline Notação & Descrição & Tipo \\
\hline $\mathcal{X}$ & Níveis de latitude dos cubos & Conjunto \\
\hline $\mathcal{Y}$ & Níveis de longitude dos cubos & Conjunto \\
\hline $\mathcal{Z}$ & Níveis de altitude dos cubos & Conjunto \\
\hline $\mathcal{I}_{x, y, z}$ & Interesse do cubo de coordenadas $x, y, z$ para o posicionamento de um totem & Parâmetro \\
\hline $\mathcal{N}$ & Número máximo de totens disponíveis & Parâmetro \\
\hline $\mathcal{A}_{x, y, z}$ & Total de cubos dentro do alcance da região de cobertura de um totem nas coordenadas $x, y, z$ & Parâmetro \\
\hline$l_{x}, l_{y}, l_{z}$ & Alcance em número de cubos do raio de transmissão de um totem nos eixos x, y e z, respectivamente & Parâmetro \\
\hline $\mathcal{R}$ & Número mínimo de totens vizinhos que devem estar dentro da região de cobertura de um totem & Parâmetro \\
\hline$c_{x, y, z}$ & Variável binária indicando se o cubo de coordenadas $x, y, z$ pertence à região coberta por algum totem & Variável \\
\hline$t_{x, y, z}$ & Variável binária indicando se há um totem posicionado no cubo de coordenadas $x, y, z$ & Variável \\
\hline
\end{tabular}

\section{Resultados}

A partir dos dados de entrada, com as informações da trilha e o alcance medido para os módulos LoRa e ZigBee, o problema de otimização formulado é executado através do solver CPLEX. Os resultados apresentados possuem uma margem de 5\% do valor da solução ótima. Essa tolerância é usada para obter soluções satisfatórias em menos tempo, sem exaurir os recursos computacionais da máquina.

\subsection{Parâmetros de Avaliação}

Como o segundo trecho da trilha requer maior cuidado por parte dos visitantes, o interesse de cada cubo pertencente a esse trecho é igual a 1. Já os cubos dos outros dois trechos da trilha possuem interesse igual a 0,5. Esses valores podem ser ajustados conforme a necessidade do problema. A conectividade entre os totens é garantida ao usar $\mathcal{R}=2$, forçando que haja sempre dois totens dentro da região de cobertura de cada totem. Assim, cada totem pode receber dados de um segundo totem e encaminhá-los para um terceiro totem.

O raio de transmissão é de $125 \mathrm{~m}$ para o LoRa e de $50 \mathrm{~m}$ para o ZigBee, conforme as medições realizadas no PARNASO em dias de chuva. Dessa forma, garantir a comunicação quando o alcance é reduzido pela chuva serve para garantir que também haverá comunicação em condições climáticas favoráveis. Para obter os resultados, são medidas as porcentagens do número de cubos cobertos e do interesse obtido ao prover comunicação nos totens. Essas medições são relativas ao número total de cubos na matriz e à soma do interesse de todos os cubos, respectivamente.

Para avaliar as métricas adotadas, variam-se dois parâmetros: o número de totens disponíveis e o índice de granularidade $g$. O número de totens disponíveis varia de 150 a 550 , sendo testados com um passo de 50 totens de uma execução para a outra. O número de totens é um dos parâmetros de avaliação porque representa custo, o que limita a aquisição dos módulos. Já o índice de granularidade é importante para refinar a precisão com a 
qual são definidas as coordenadas para o posicionamento dos totens. Esse índice é um número inteiro positivo usado para ajustar o tamanho do cubo. Um índice de granularidade maior e, portanto, uma granularidade mais fina, resulta em uma localização mais precisa. Entretanto, a sua escolha envolve um trade-off entre essa precisão e tanto o tempo de execução quanto a capacidade computacional necessária para executar a otimização.

Para definir o índice de granularidade, primeiro é necessário notar que existe um limite máximo para o tamanho do cubo. A partir da Inequação 5, é necessário que um totem alcance, pelo menos, os seus cubos vizinhos, por aresta e por face. Dessa forma, a região de cobertura do totem terá cubos o suficiente para o posicionamento de outros totens, o que é necessário para que estejam conectados. Isso faz com que, no mínimo, $l_{x}=l_{y}=l_{z}=1$. Para garantir isso, o lado máximo do cubo $\left(L C_{\max }\right)$ é dado por:

$$
L C_{\max }=\frac{2 \cdot R T X}{2 \cdot l_{\min }+1} .
$$

Como $l_{\text {min }}=1$ nas três direções, o totem deve cobrir, no mínimo, o próprio cubo, o anterior e o posterior. Então, fazendo $l_{\min }=1$, o cubo deve ter, no máximo, dois terços do raio de transmissão $(R T X)$. Uma vez que o $R T X$ para o ZigBee é inferior ao do LoRa, o $L C_{\max }$ adotado para os experimentos é referente ao ZigBee, cujo $R T X$ obtido nas medições de campo é de $50 \mathrm{~m}$. Com isso, tem-se da Equação 6 que $L C_{\max }=33,3 \mathrm{~m}$. Isso é feito de modo a tornar possível a comparação entre as tecnologias com os mesmos valores de lado do cubo.

Para testar tamanhos menores de cubo, tornando a matriz mais densa e a granularidade mais fina, o índice de granularidade $g$ é usado na Equação 7. O índice é um número inteiro pelo qual $R T X$ é dividido, obedecendo o limite máximo do lado do cubo, conforme definido pela Equação 6. Para o experimento, são considerados $g=3$ e $g=4$, que fornecem os lados de cubo $33,3 \mathrm{~m}$ e $25 \mathrm{~m}$, respectivamente.

$$
L C_{g}=\frac{2 \cdot R T X}{g} \leq L C_{\max }
$$

\subsection{Cenário Ilimitado}

Para validar a formulação que envolve a maximização do interesse para um dado número $\mathcal{N}$ de totens disponíveis, também é simulado um cenário ideal, com infinitos totens disponíveis e tendo como função objetivo minimizar o número de totens posicionados. Para o cenário ilimitado, a Equação 2 é substituída pela Equação 8. Já a restrição de obediência ao número de totens disponíveis, da Inequação 3, é substituída pela Inequação 9. Essa nova restrição garante que todos os cubos de interesse devem ser iluminados por algum totem, independente de quantos totens são necessários para isso. Se um cubo for de interesse nulo, ele pode ser iluminado ou não, o valor não importa, representado pela notação $\boldsymbol{*}$, ou seja, don't care. As demais restrições são mantidas. Os resultados do cenário ilimitado correspondem à cobertura de $100 \%$ dos cubos e obtenção de $100 \%$ do interesse do cenário de teste.

$$
\operatorname{minimizar} \sum_{x \in \mathcal{X}} \sum_{y \in \mathcal{Y}} \sum_{z \in \mathcal{Z}} t_{x, y, z}
$$




$$
\text { sujeito a } \quad c_{x, y, z}=\left\{\begin{array}{ll}
1, & \text { if } \mathcal{I}_{x, y, z}>0 \\
\boldsymbol{x}, & \text { if } \mathcal{I}_{x, y, z}=0
\end{array} \quad \forall m \in \mathcal{M}, q \in \mathcal{Q}\right.
$$

\subsection{Cenário de Teste}

A Figura 6 mostra que, a partir de 200 totens, o uso do LoRa já permite cobrir todos os cubos da travessia, ao passo que os totens ZigBee cobrem até $60 \%$ dos cubos nesse ponto. A quantidade de totens ZigBee necessária para uma cobertura de $100 \%$ é quase três vezes maior do que a necessária para o LoRa. Por exemplo, são necessários 550 totens ZigBee (com g = 3) para cobrir o máximo de cubos. A taxa de crescimento das curvas possui uma tendência linear, visto que iluminar cada cubo contribui da mesma forma em termos de número de cubos cobertos. Como o alcance da transmissão do módulo LoRa é mais de duas vezes maior que o alcance do ZigBee, são necessários menos totens LoRa para iluminar os cubos e, consequentemente, obter maiores valores de interesse.

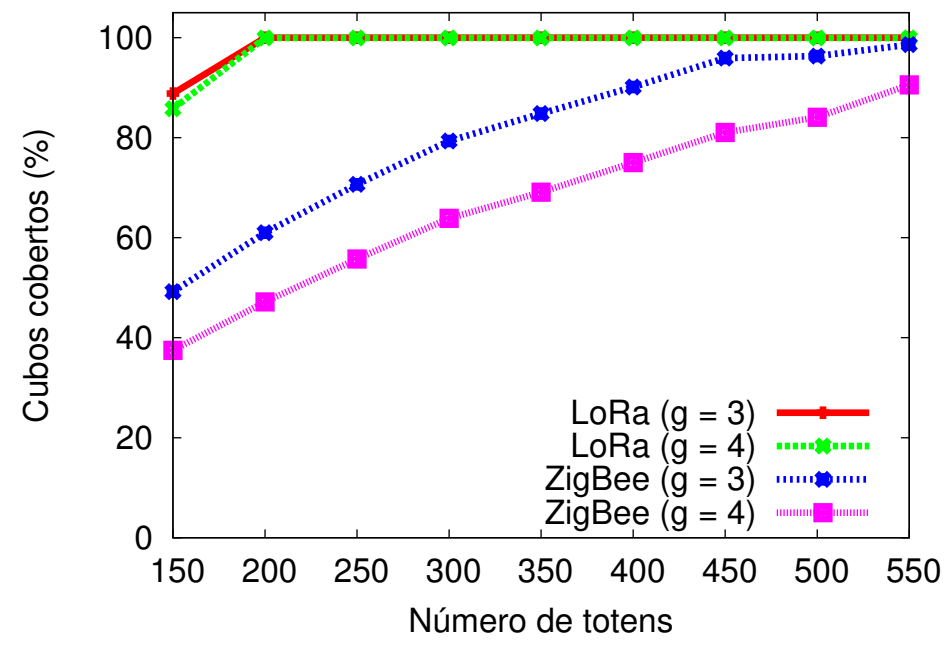

Figura 6. Cobertura em cubos para totens LoRa e ZigBee com diferentes granularidades.

Ao tornar a granularidade mais fina, o problema passa a ter mais cubos e os resultados se tornam mais precisos. Isso diminui os valores obtidos para cobertura e interesse, pois os cubos maiores mascaram esses resultados, jogando os valores para cima. Isso é coerente porque, se um pequeno trecho da trilha atravessa um cubo maior, ele é considerado iluminado como um todo. No entanto, ao dividir esse cubo em quatro cubos menores, por exemplo, se esse mesmo trecho estiver contido em apenas um dos quatro cubos menores, apenas esse será considerado iluminado, diminuindo a porcentagem de cubos cobertos.

Além da porcentagem de cubos cobertos, o interesse obtido ao cobrir esses cubos também é medido, conforme mostra a Figura 7. Claramente, quanto mais totens, maior a cobertura e o interesse obtido, um aspecto bastante direto ao comparar as Figuras 6 e 7. A taxa de crescimento para a Figura 7 mostra uma diferença importante, embora sutil, ela diminui conforme a quantidade de totens disponíveis aumenta. Isso ocorre porque o objetivo é maximizar o interesse no cenário de teste, o que inclina o CPLEX a cobrir primeiro os cubos mais interessantes, que são os do meio da trilha. Assim, o interesse 
aumenta em 1 para cada cubo nesse trecho, mas, quando todo o trecho central é coberto, o interesse passa a aumentar em 0,5 para cada novo cubo coberto.

A Figura 7 também confirma o que é observado na Figura 6 pois, se a cobertura é de $100 \%$ de 200 totens LoRa em diante, todo o interesse dos cubos foi obtido, estando também em 100\%, o que significa que o interesse obtido é igual ao somatório do interesse de todos os cubos do problema. Como o trecho central é mais significativo em termos de interesse, é possível ver, por exemplo, que metade dos cubos cobertos ao usar ZigBee com $g=3$ já são responsáveis por mais de $70 \%$ do interesse da trilha.

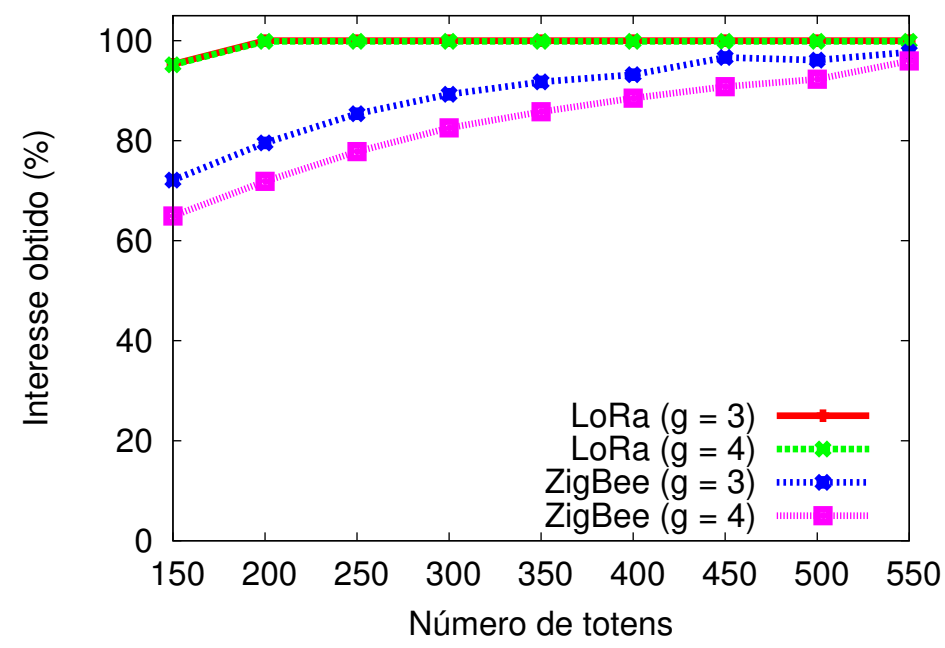

Figura 7. Interesse obtido para totens LoRa e ZigBee com diferentes granularidades.

\section{Trabalhos Relacionados}

Vários trabalhos propõem algoritmos para solucionar problemas de cobertura, alguns deles, na área de redes de sensores. Em [Huang and Tseng 2005], é feito um survey desse tema. Em [Zhu et al. 2012], também é apresentado um levantamento que classifica o problema de cobertura de diferentes pontos de vista, analisando inclusive a relação entre cobertura e conectividade. O trabalho de [Van Le et al. 2016] apresenta uma formulação de ILP para o posicionamento de nós em uma rede de sensores móveis, maximizando a cobertura em um problema com restrições de conectividade. São propostas três heurísticas para a solução do problema. Os resultados das simulações de Van Le et al. mostram que o algoritmo obtém bons resultados para eficiência de cobertura e uso de energia.

Também são trabalhos relacionados a este artigos que investigam sistemas de geolocalização. Um sistema capaz de transmitir dados de posicionamento usando LPWAN em ambientes outdoor é desenvolvido em [Carbonés Fargas 2017], demostrando ser factível a localização de um terminal estático com uma precisão de 100 metros. Já em [Baharudin and Yan 2016], é proposta uma solução híbrida: uma rede de sensores de longo alcance para rastrear objetos móveis. Múltiplos sensores movem-se a partir de uma estação base e suas informações de geolocalização são obtidas via GPS e enviadas usando módulos LoRa. [Lima et al. 2016] busca resolver o problema de geolocalização em redes de sensores usando o nível de recepção do sinal para estimar as posições dos nós. 
O presente trabalho se diferencia desses trabalhos relacionados por utilizar a geolocalização por LoRa e ZigBee com múltiplos saltos dentro do ambiente de floresta, empregando os totens de comunicação. A análise do posicionamento dos totens e a sua formulação através de um problema de ILP bem como a a modelagem geométrica para diminuir o tamanho do problema são contribuições originais deste trabalho.

\section{Conclusão e Trabalhos Futuros}

Este artigo investigou o posicionamento de pontos de acesso sem-fio, denominados de totens, em regiões de floresta para fornecer comunicação e melhorar a segurança de visitantes em parques florestais. Esse problema é inspirado em uma aplicação real em desenvolvimento no Parque Nacional da Serra dos Órgãos. A abordagem inova ao formar uma matriz tridimensional com os dados geográficos da região. Assim, considera-se a diferença de altitude nos percursos da travessia para posicionar os totens. A partir dos dados de alcance de transmissão obtidos em medições locais, um problema de ILP é formulado e resolvido através do solver CPLEX.

Através da solução do problema de otimização, é possível concluir que a modelagem geométrica permite a cobertura dos cubos da trilha, de acordo com parâmetros de interesse. No caso estudado, o parâmetro de interesse prioriza o posicionamento de totens no segundo trecho da Travessia Petrópolis-Teresópolis, considerado o de maior risco. Foram analisadas duas tecnologias de comunicação, o LoRa e o ZigBee, com os dados obtidos em medições feitas no próprio parque. Como o raio de transmissão obtido com LoRa é maior que o do ZigBee, são necessários menos totens para cobrir a trilha. Com 200 totens LoRa, já se obtém uma cobertura de 100\%. Para o ZigBee, isso só é possível a partir de 550 totens. Também são utilizados diferentes tamanhos de cubo para variar a granularidade da solução, obtendo resultados com valores próximos.

Como trabalhos futuros, novos experimentos serão realizados para novos módulos de tecnologias diferentes, como Bluetooth Low Energy e IEEE 802.11ah, mais adequadas para o uso no modo interativo do sistema de monitoração. Também serão consideradas novas métricas, como a vazão, a latência e a taxa de perda de pacotes, para comparar as diferentes tecnologias. Como o ZigBee possui taxas nominais superiores ao LoRa, esperase que seu desempenho seja superior para essas métricas. Sabendo que os problemas de ILP são conhecidamente NP-difíceis, sua escalabilidade é limitada. Assim, entre os trabalhos em andamento está ainda a investigação de algoritmos para soluções através de heurísticas, para lidar com valores maiores de $g$ e regiões geográficas mais extensas, sem depender de um solver ou impactar nos tempos de execução.

\section{Agradecimentos}

Este trabalho foi parcialmente financiado pelas agências de fomento CAPES, CNPq, FAPERJ e pelos processos $n^{\circ} 15 / 24494-8$ e $n^{\circ}$ 15/24490-2, da FAPESP. Os autores estendem seus agradecimentos ao ICMBio, ao PARNASO, especialmente ao seu Coordenador de Conservação e Manejo da Biodiversidade, Jorge "Julião"Nascimento, e a Adam Schneider, criador do GPS Visualizer. 


\section{Referências}

Baharudin, A. M. and Yan, W. (2016). Long-range wireless sensor networks for geolocation tracking: Design and evaluation. In Electronics Symposium (IES), 2016 International, pages 76-80. IEEE.

Bor, M., Vidler, J., and Roedig, U. (2016). Lora for the internet of things. In Proceedings of the 2016 International Conference on Embedded Wireless Systems and Networks, EWSN '16, pages 361-366, USA. Junction Publishing.

Carbonés Fargas, B. (2017). IoT and tracking capabilities in LPWANs. Master's thesis, Universitat Politècnica de Catalunya.

Figueiredo, C. M. S., Nakamura, E. F., Ribas, A. D., de Souza, T. R. B., and Barreto, R. S. (2009). Assessing the communication performance of wireless sensor networks in rainforests. In 2009 2nd IFIP Wireless Days (WD), pages 1-6.

Huang, C.-F. and Tseng, Y.-C. (2005). The coverage problem in a wireless sensor network. Mob. Netw. Appl., 10(4):519-528.

Liao, C.-H., Zhu, G., Kuwabara, D., Suzuki, M., and Morikawa, H. (2017). Multi-hop LoRa networks enabled by concurrent transmission. IEEE Access, 5:21430-21446.

Lima, M. M., Oliveira, H. A. B. F. d., Nakamura, E. F., Barreto, R., and Guidoni, D. L. (2016). Agregação de dados com desvio de buracos para redes de sensores sem fio com sinks de alto alcance. In Anais do XXXIV Simpósio Brasileiro de Redes de Computadores e Sistemas Distribuıdos - SBRC 2016, pages 614-629. SBRC.

Loureiro, A. A. F., Nogueira, J. M. S., Ruiz, L. B., Mini, R. A. d. F., Nakamura, E. F., and Figueiredo, C. M. S. (2003). Redes de sensores sem fio. In Minicursos de XXI Simpósio Brasileiro de Redes de Computadores (SBRC'2003), pages 179-226.

Marais, J. M., Malekian, R., and Abu-Mahfouz, A. M. (2017). LoRa and LoRaWAN testbeds: A review. In AFRICON, 2017 IEEE, pages 1496-1501. IEEE.

Semtech Corporation (2012). Zigbee Specification. http://www.zigbee.org/ download/standards-zigbee-specification.

Semtech Corporation (2015). Lora ${ }^{\mathrm{TM}}$ modulation basics an1200.22. https://www . semtech.com/uploads/documents/an1200.22.pdf.

Stutzman, W. L. and Thiele, G. A. (2012). Antenna theory and design. John Wiley \& Sons, 3rd edition.

Van de Velde, B. (2017). LoRa forwarder. https://github.com/texus/ LoRaWAN-forwarder.

Van Le, D., Oh, H., and Yoon, S. (2016). Environment learning-based coverage maximization with connectivity constraints in mobile sensor networks. IEEE Sensors Journal, 16(10):3958-3971.

Zhu, C., Zheng, C., Shu, L., and Han, G. (2012). A survey on coverage and connectivity issues in wireless sensor networks. Journal of Network and Computer Applications, 35(2):619-632. 